\title{
Singularities and topologically protected states in twisted bilayer graphene
}

Cite as: Appl. Phys. Lett. 116, 011602 (2020); https://doi.org/10.1063/1.5135071

Submitted: 05 November 2019 . Accepted: 26 December 2019 . Published Online: 08 January 2020

Qirong Yao, Xingchen Chen, Rik van Bremen, Kai Sotthewes (D), and Harold J. W. Zandvliet (D)
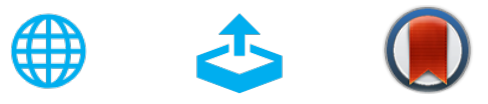

View Online

\section{ARTICLES YOU MAY BE INTERESTED IN}

Detection of spin-orbit torque with spin rotation symmetry

Applied Physics Letters 116, 012404 (2020); https://doi.org/10.1063/1.5129548

Sensing anisotropic stresses with ferromagnetic nanowires

Applied Physics Letters 116, 013104 (2020); https://doi.org/10.1063/1.5132539

Long-range coupling interaction between a non-magnetic transition metal capping layer and a neighboring magnetic layer

Applied Physics Letters 116, 012405 (2020); https://doi.org/10.1063/1.5133652

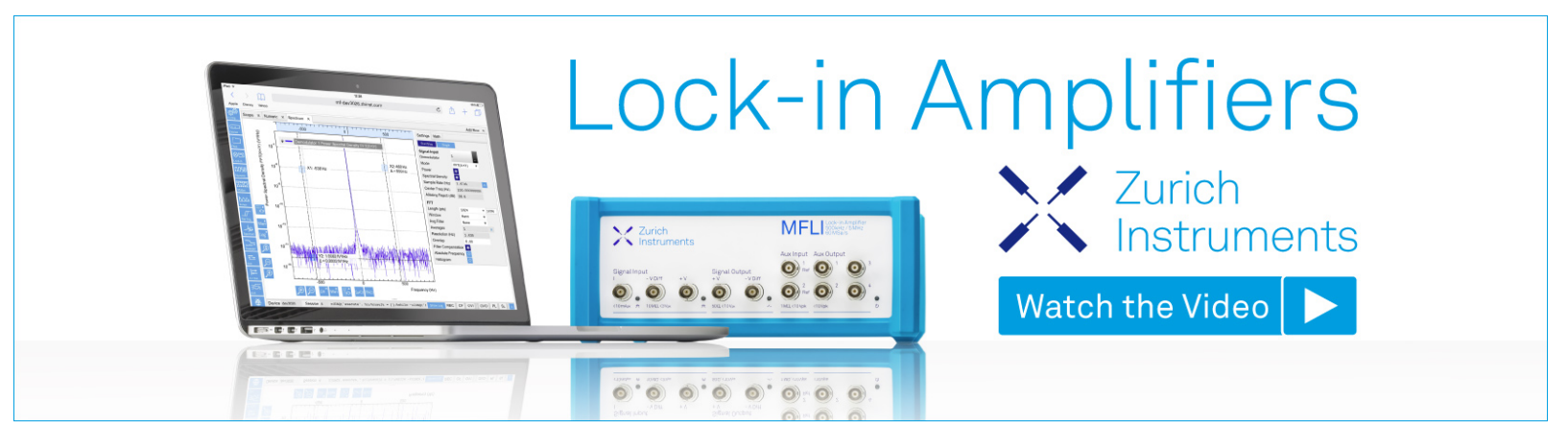




\title{
Singularities and topologically protected states in twisted bilayer graphene
}

\author{
Cite as: Appl. Phys. Lett. 116, 011602 (2020); doi: 10.1063/1.5135071 \\ Submitted: 5 November 2019 . Accepted: 26 December 2019 . \\ Published Online: 8 January 2020
}

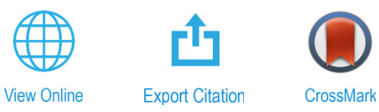

Qirong Yao, ${ }^{\text {a) }}$ Xingchen Chen, Rik van Bremen, Kai Sotthewes, (D) and Harold J. W. Zandvliet

\begin{abstract}
AFFILIATIONS
Physics of Interfaces and Nanomaterials, MESA+ Institute for Nanotechnology, University of Twente, P.O. Box 217, 7500 AE Enschede, The Netherlands
\end{abstract}

a) Author to whom correspondence should be addressed: q.yao@utwente.nl

\begin{abstract}
We have studied the structural and electronic properties of twisted bilayer graphene by scanning tunneling microscopy (STM). For twist angles in the range of about $1^{\circ}$ to $4^{\circ}$, the twisted bilayer graphene possesses two Van Hove singularities in the vicinity of the Fermi level. We use the exact location of these Van Hove singularities to determine the twist angle dependent interlayer hopping energy. For a twist angle of $0.6^{\circ}$, we found a hexagonal network of topologically protected one-dimensional channels that run along the boundaries of the $\mathrm{AB} / \mathrm{BA}$ domains. The electric field in the tunnel junction is responsible for the breaking of the symmetry of the AB and BA domains and the development of the hexagonal network of topologically protected states. The latter shows that the electric field in the tunneling junction can significantly affect the topological nature of two-dimensional materials, and therefore, one should be cautious when interpreting scanning tunneling microscopy and spectroscopy experiments of this class of materials.
\end{abstract}

Published under license by AIP Publishing. https://doi.org/10.1063/1.5135071

If two lattices, which are slightly twisted with respect to each other, are placed on top of each other, a superstructure is found, which is referred to as a moiré structure. In particular, twisted bilayers of graphene have received quite some attention during the last few years owing to the rich variety of fascinating physical properties that they exhibit as a function of twist angle. ${ }^{1-6}$ The periodicity, $\lambda$, of the twisted bilayer graphene is $\lambda=\mathrm{a} / 2 \sin (\theta / 2)$, where $a=0.246 \mathrm{~nm}$ is the lattice constant of graphene and $\theta$ the twist angle. A twist angle of $\theta=0^{\circ}$ corresponds to the normal $\mathrm{AB}$ stacking, also referred to as the Bernal stacking, while an angle of $\theta=60^{\circ}$ corresponds to the AA stacking. At a twist angle of exactly $30^{\circ}$, a two-dimensional quasicrystal with a 12 fold rotational order, but no translation order, forms. Interestingly, not only the size of the unit cell but also the electronic structure of twisted bilayer graphene depends on the exact value of the twist angle. For a twist angle in the range of about $1^{\circ}$ to a few degrees, bilayer graphene exhibits two electronic states in the vicinity of the Fermi level. These electronic states, which form due to the crossing of the Dirac cones of both graphene sheets, have an appreciable density of states and can be brought arbitrarily close to the Fermi level, leading to electronic instabilities and correlated electron phases, such as superconductivity, 4,8 mott metal-insulator transition and/or Wigner crystallization, ${ }^{9,10}$ and tunable magnetism. ${ }^{11,12}$ For an angle of $\sim 1.1^{\circ}$, the so-called magic angle, the energy bands are completely flat at the
Fermi level, resulting in a vanishing Fermi velocity. Via electrostatic doping, Cao et al. managed to convert a $1.1^{\circ}$ twisted bilayer graphene from an insulator to a superconductor. ${ }^{4}$ As pointed out by Bistritzer and MacDonald, there are more "magic" angles at which the Fermi velocity vanishes. ${ }^{13}$ For angles smaller than about $1^{\circ}$, another interesting phenomenon has been reported. ${ }^{14,15}$ The twisted bilayer graphene exhibits $\mathrm{AB}$ and $\mathrm{BA}$ stacked configurations. The application of an external electric field breaks the inversion symmetry of these two stacking configurations. The valley Chern number, i.e., the integral of the momentum space Berry curvature over a single valley, is +1 or -1 with a sign that depends on the stacking configuration $(\mathrm{AB}$ or $\mathrm{BA})$ and the direction of the electric field. Owing to this sign change of the Chern number in going from an $\mathrm{AB}$ region to a $\mathrm{BA}$ region, the domain wall between the gapped $\mathrm{AB} / \mathrm{BA}$ regions will exhibit two counter-propagating topologically protected helical channels (one set per valley).

Here, we will focus our attention on the structural and electronic properties of small angle $\left(<4^{\circ}\right)$ twisted bilayer graphene using scanning tunneling microscopy. The exact location of the electronic states will allow us to determine the interlayer hopping energy. Of particular interest are twisted bilayer graphene (TBG) with very small twist angles $\left(<1^{\circ}\right)$, where the electronic states, the so-called Van Hove singularities, are very close to the Fermi level. 
There are several ways to produce or obtain twisted graphene layers. The most trivial one is to simply take a highly oriented pyrolytic graphene (HOPG) substrate. The graphene layers of HOPG are mainly stacked in the so-called Bernal (or AB) configuration; however, occasionally one finds, by using for instance scanning tunneling microscopy, regions where the top graphene layer is rotated with respect to the underlying HOPG substrate. HOPG is, however, not a perfect substrate for twisted bilayers of graphene for several reasons: (1) the relevant electronic states of twisted bilayer graphene, which are located in the vicinity of the Fermi level, can hybridize with the HOPG substrate owing to its semimetallic nature and (2) it is very difficult to electrostatically dope a twisted bilayer graphene on a HOPG substrate. In order to overcome these problems, we have used the dry-transfer and tear-and-stack technique to prepare bilayer graphene with a controllable twisted angle on a hexagonal boron nitride (h-BN)/ $\mathrm{SiO}_{2}(300 \mathrm{~nm}) / \mathrm{Si}$ substrate. ${ }^{4,16-19}$ First, a thin multilayer of hexagonal boron nitride (h-BN) is placed on a Si substrate that is coated with a $300 \mathrm{~nm}$ thick $\mathrm{SiO}_{2}$ film. Subsequently, a single layer flake of graphene is placed on the h-BN multilayer using a viscoelastic stamp that is mounted on an XYZ- $\theta$ stage. $\mathrm{H}-\mathrm{BN}$, also referred to as white graphene, is the ideal substrate for graphene owing to its large bandgap of about $6 \mathrm{eV}$. Second, a small part from of a single layer graphene flake is torn apart using the stamp. Before we place this flake back onto the remainder of the graphene flake, we rotate the $\mathrm{Si} / \mathrm{SiO}_{2}$ substrate by an angle $\theta$. We have used an optical microscope from Leica (DM2500MH), and the Raman map and spectra were recorded using a WITec alpha 300R Raman microscope, with a $532 \mathrm{~nm}$ laser. The highly oriented pyrolytic graphite (HOPG), natural graphite, and h$\mathrm{BN}$ crystals were purchased from HQ Graphene (Groningen, The Netherlands). Further details of fabrication can found in the supplementary material. The scanning tunneling microscopy (STM) and scanning tunneling spectroscopy (STS) data were recorded at room temperature using an ultrahigh vacuum Omicron scanning tunneling microscope. We have used electrochemically etched W-tips for our STM and STS experiments. The base pressure of the ultrahigh vacuum system is $3 \times 10^{-11}$ mbar.

The structural and electronic properties of the single layer and bilayer graphene samples that have been placed on the h-BN substrates have been investigated using STM and STS. As we will show later, we have found regions with different twist angles in all our twisted bilayer graphene (TBG) samples.

In Fig. 1(a), an optical image and a Raman map of a monolayer graphene are displayed in panels I and II, respectively. The graphene flake was deposited onto the $\mathrm{SiO}_{2} / \mathrm{Si}$ substrate by mechanical exfoliation. The Raman spectrum shown in Fig. 1(b) reveals that we are
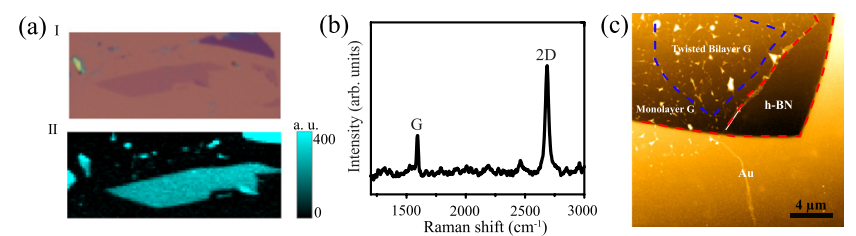

FIG. 1. (a) Panel I: mechanically exfoliated monolayer graphene on $\mathrm{SiO}_{2}(300 \mathrm{~nm}) / \mathrm{Si}$ substrate; panel II: Raman map of the graphene flake shown in panel I. (b) Raman spectroscopy of the monolayer graphene in panel I. (c) Atomic force microscopy image of a twisted bilayer graphene on the h-BN substrate with the Au electrode. dealing with a monolayer graphene. The spectrum has two pronounced peaks corresponding to the $\mathrm{G}$ mode and the $2 \mathrm{D}$ mode, respectively. The $\mathrm{G}$ mode is found at about $1585 \mathrm{~cm}^{-1}$, which suggests a slight doping of the graphene layer. This effect is typically observed for graphene on $\mathrm{SiO}_{2}$. The fact that the intensity of the $2 \mathrm{D}$ peak is substantially higher than the intensity of the $G$ peak indicates that we are dealing with a single layer of graphene. ${ }^{20}$ Figure 1 (c) shows an atomic force microscopy (AFM) image of the twisted bilayer graphene sample that we prepared for the STM and STS measurements. In the AFM image, we have shown the pristine h-BN, the monolayer graphene/h$\mathrm{BN}$, and twisted bilayer graphene/h-BN. In order to be able to perform STM measurements, we have evaporated an $\mathrm{Au} / \mathrm{Cr}$ electrode on our sample using a shadow mask.

In Fig. 2(a), a scanning tunneling microscopy image of single layer graphene on hexagonal boron nitride (h-BN) is shown. The periodicity of a single graphene on h-BN is given by $\frac{(1+\delta) a}{\sqrt{2(1+\delta)(1-\cos \theta)+\delta^{2}}}, 21$ where $\delta$ is the lattice mismatch of graphene and h-BN, $a$ the lattice constant of graphene, and $\theta$ the twist angle between the graphene layer and the h-BN substrate. From Fig. 2(a), we extract a moiré periodicity of about $12 \mathrm{~nm}$, which corresponds to a rotational angle of $\sim 0.7^{\circ}$. In Fig. 2(b), a scanning tunneling spectrum in the bias range of $-0.5 \mathrm{~V}$ to $0.5 \mathrm{~V}$ is displayed. The differential conductivity, $d \mathrm{I} / d \mathrm{~V}$, which is proportional to the density of the graphene layer, exhibits a well-defined $\mathrm{V}$-shape. This $\mathrm{V}$-shape reveals that we are dealing with a twodimensional Dirac material that is electronically decoupled from its underlying h-BN substrate. The graphene layer on the h-BN substrate is slightly $p$-doped.

In Fig. 3, two scanning tunneling microscopy images of twisted bilayer graphene on $\mathrm{h}-\mathrm{BN}$ recorded in two different regions of the same flake are shown. Although we can rotate our stamp with a precision better than $0.1^{\circ}$, we always observed the coexistence of regions with different twist angles. The difference in the twist angle is sometimes as large as $1^{\circ}$ to $2^{\circ}$; however, we have also made twisted bilayer graphene samples where the variation in the twist angle is substantially smaller. Based on these observations, we have to conclude that the tear and stack method does not result in a single and well-defined twist angle. The coexistence of regions with different twist angles is most probably caused by winkles and blisters. The formation of these winkles and blisters is virtually unavoidable during the stacking process; see Fig. 1(c) for an AFM image that shows several wrinkles and blisters. In many cases, transport studies of these twisted bilayers are
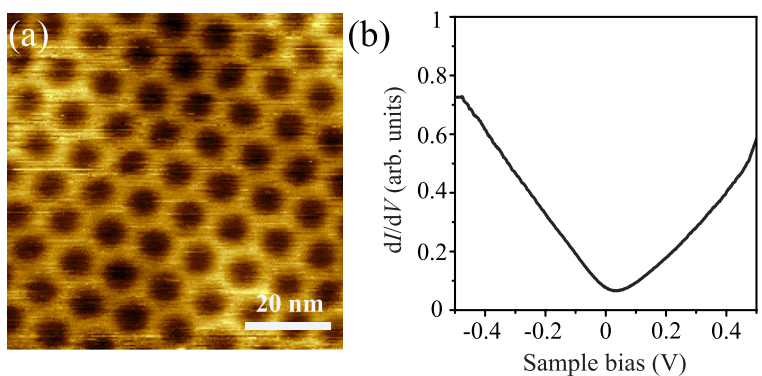

FIG. 2. Scanning tunneling microscopy image (a) and scanning tunneling spectroscopy spectrum (b) of single layer graphene on an h-BN substrate, respectively. The sample bias is $-0.5 \mathrm{~V}$, and the tunneling current is $200 \mathrm{pA}$. 
(a)

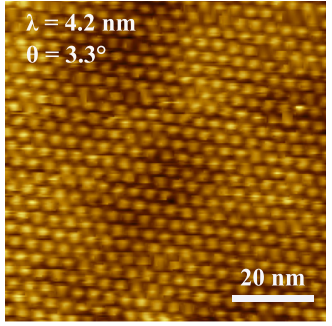

(b)

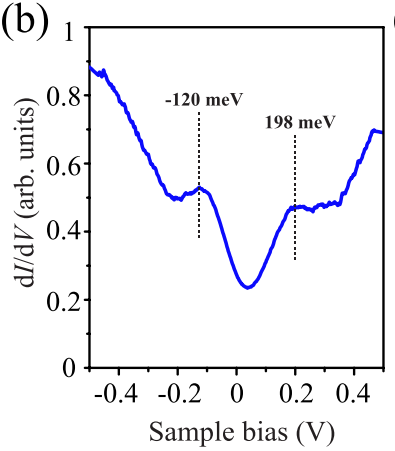

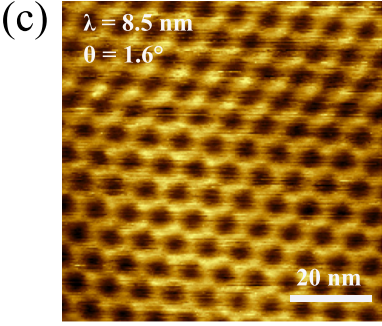

(d)

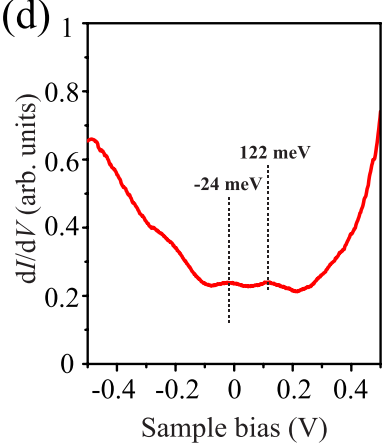

FIG. 3. Scanning tunneling microscopy images and scanning tunneling spectroscopy spectrum of twisted bilayer graphene on an h-BN substrate, respectively. (a) and (c) The sample bias is $0.4 \mathrm{~V}$, and the tunneling current is $0.5 \mathrm{nA}$. (b) and (d) Set points are a sample bias of $-0.5 \mathrm{~V}$ and a tunnel current of $200 \mathrm{pA}$.

performed using electrical contacts that are deposited onto the twisted bilayer. The electrical contacts are typically positioned a few micrometers apart, and one often assumes that the twist angle is constant in the area between the electrical contacts. Our experiments reveal that, however, this is usually not the case. This experimental finding is of great relevance for the interpretation of macroscopic transport measurements.

We have selected regions on the same twisted bilayer graphene, which exhibit a well-defined moiré pattern (see Fig. 3). In order to

compare the images with each other, we have used the same sample bias $(0.4 \mathrm{~V})$ and tunneling current $(0.5 \mathrm{nA})$. The bright dots in Figs. $3(\mathrm{a})$ and $3(\mathrm{c})$ are the AA stacked regions. The moire patterns have a periodicity of $4.2 \mathrm{~nm}$ and $8.5 \mathrm{~nm}$, corresponding to twist angles of $3.3^{\circ}$ and $1.6^{\circ}$, respectively. STS spectra reveal two well-pronounced peaks, which are outlined by the dashed lines in Figs. 3(b) and 3(d). The peaks, which are referred to as Van Hove singularities (VHs), arise due to the hybridization of the Dirac cones of the top and bottom graphene layer. ${ }^{22}$ Using the continuum approximation for a bilayer graphene with a small twist angle, the energy gap of the two Van Hove singularities is given by $\Delta E_{V H s}=2 \hbar v_{F} K \sin (\theta / 2)-2 \tilde{t}_{\perp}$, where $v_{F}$ is the Fermi velocity of the twisted graphene bilayer and $\tilde{t}_{\perp}$ the interlayer hopping parameter. ${ }^{23}$ It is clear from this expression that the gap between the two VHs is controlled by the twist angle, which is also supported by our experimental results. The $\Delta E_{V H s}$ value is $318 \mathrm{meV}$ for $\theta=3.3^{\circ}, 250 \mathrm{meV}$ for $\theta=2.0^{\circ}$ (not shown here), and $146 \mathrm{meV}$ for $\theta=1.6^{\circ}$. Assuming a Fermi velocity of $\sim 10^{6} \mathrm{~m} / \mathrm{s}$, we determined a value for the interlayer hopping energy $\tilde{t}_{\perp}$ of $160 \mathrm{meV}$ for $\theta=3.3^{\circ}$, $130 \mathrm{meV}$ for $\theta=2.0^{\circ}$, and $80 \mathrm{meV}$ for $\theta=1.6^{\circ}$, respectively.

In Fig. 4(a), an STM image of a twisted bilayer graphene with a twist angle of $0.6^{\circ}$ is shown. Interestingly, Fig. 4 (a) does not show the aforementioned bright dots, but rather a hexagonal pattern consisting of bright lines. Figure 4(b) shows an atomically resolved small scale image of the $\mathrm{AA}, \mathrm{AB}$, and $\mathrm{BA}$ stacked regions. The $\mathrm{AA}$ stacked region reveals a honeycomb structure, which is outlined in green. For the $A B$ and BA stacked regions, one of the triangular sublattices shows up more pronounced than the other sublattice (outlined in magenta and blue). The STM images of AB and BA regions are very comparable to STM images of Bernal stacked highly oriented graphite substrates. The bright lines in Fig. 4(a) correspond to the domain boundaries between the $\mathrm{AB}$ and $\mathrm{BA}$ stacked regions. We suggest that these bright lines are topologically protected one-dimensional conducting channels that emerge because the electric field in the tunneling junction breaks the inversion symmetry between the $\mathrm{AB}$ and $\mathrm{BA}$ stacked regions. ${ }^{15,24-26}$ The electric field in the scanning tunneling microscopy junction has two components. One component is due to the difference in the work
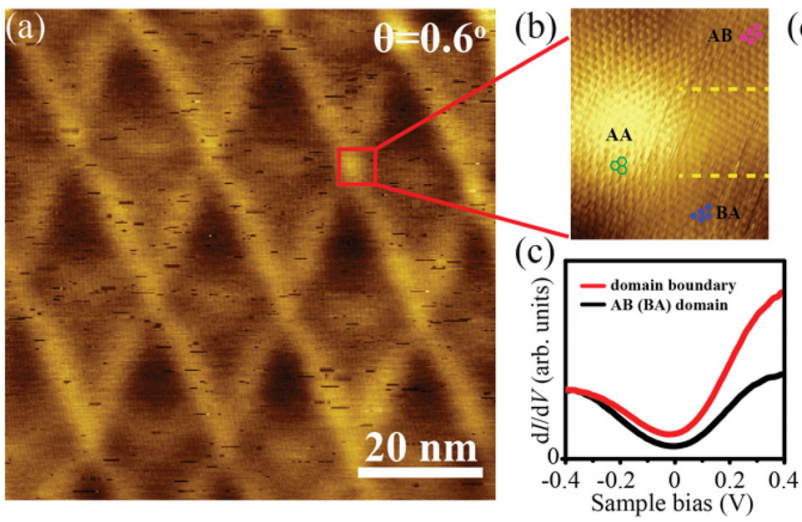

(d)

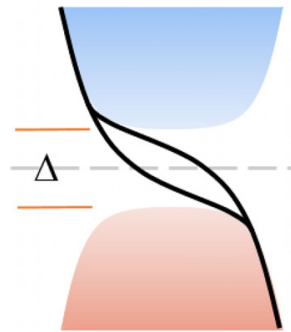

$\mathrm{K}$

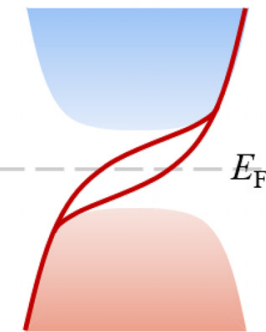

$\mathrm{K}^{\prime}$

FIG. 4. (a) Large-scale scanning tunneling microscopy image $(70 \mathrm{~nm}$ by $70 \mathrm{~nm})$ of a twisted bilayer graphene with a twist angle of $0.6^{\circ}$. (b) Atomically resolved small scale image revealing the structure of the $A A, A B$, and $B A$ stacked regions. The honeycomb $(A A)$ and triangular lattices $(A B$ and $B A)$ are outlined in green, magenta, and blue, respectively. The sample bias is $0.4 \mathrm{~V}$, and the tunneling current is $1 \mathrm{nA}$. (c) Differential conductivity (d//dV) recorded on the domain boundaries (red) and the $A B$ and $B A$ stacked domains (black). Set points: the sample bias and tunnel current are $-0.6 \mathrm{~V}$ and $1.0 \mathrm{nA}$, respectively. (d) Electronic band structure of twisted bilayer graphene with an $\mathrm{AB} / \mathrm{BA}$ domain boundary. There are two topologically protected one-dimensional states per valley. $\Delta$ and $E_{F}$ refer to the bandgap and Fermi level, respectively. 
function of the substrate and scanning tunneling microscopy tip, and the other component originates from the applied voltage difference between the substrate and the tip. It is important to point out that the work function of both graphene and $\mathrm{W}$ tip is about $4.5 \mathrm{eV}$, and therefore, the electric field component related to the difference in the work function between the substrate and the tip is quite small here. The valley Chern number of the $\mathrm{AB}$ and $\mathrm{BA}$ stacked domains is either +1 or -1 depending on the stacking order and direction of the electric field; one expects to find, in total, four topologically protected channels per domain boundary [see Fig. 4(d) for a schematic diagram of the electronic structure of bilayer graphene with an $\mathrm{AB} / \mathrm{BA}$ domain boundary]. The factor of four comes from the spin and valley degeneracy. Since the valley Chern numbers change from +1 to -1 and from -1 to +1 , respectively, the total number of conducting channels is 4 , resulting in a conductance of $4 e^{2} / h$. The development of these topologically protected one-dimensional channels has already been observed by Martin et al. ${ }^{24}$ by electrostatic lateral confinement. Several years later, Ju et al..$^{25}$ and Yin et al. ${ }^{26}$ reported the presence of these topologically protected one-dimensional channels on stacking fault domain boundaries, which are occasionally found on Bernal stacked graphene bilayers. It should be pointed out here that our observation of a network of topologically protected one-dimensional channels is very comparable to the findings of Huang et al. ${ }^{15}$ Huang et al. ${ }^{15}$ also made use of a twisted bilayer graphene on an h-BN substrate. However, these authors used a back-gate electrode to apply an external electrical field, whereas we used the electric field of the tunnel junction.

In summary, we have shown that the tear-and-stack method, which is frequently used to prepare well-defined twisted bilayers of graphene on h-BN substrates, results in a sample that exhibits regions with different twist angles. The coexistence of the domains with different twist angles is caused by the formation of wrinkles and blisters during the tear-and-stack process. Besides the structural properties, we have also studied the electronic properties of the twisted bilayer graphene samples. Twisted bilayers graphene on h-BN with twist angles in the range of $1^{\circ}$ to $4^{\circ}$ possess two Van Hove singularities in the vicinity of the Fermi level. The location of these Van Hove singularities allows us to determine the interlayer hopping energy. Scanning tunneling microscopy measurements reveal that small angle $\left(<1^{\circ}\right)$ twisted bilayer graphene exhibits a hexagonal pattern of topologically protected one-dimensional conducting channels. The electric field in the tunnel junction is responsible for the breaking of the symmetry of the $\mathrm{AB}$ and $\mathrm{BA}$ domains and the development of the hexagonal network of topologically protected states. The latter shows that one should be very careful when interpreting scanning tunneling microscopy and spectroscopy experiments of two-dimensional materials.

See the supplementary material for the details of the preparation method of twisted bilayer graphene on hexagonal boron nitride.

This work was part of the research program on 2D semiconductor crystals with Project No. FV157-TWOD, which was financed by the Netherlands Organization for Scientific Research (NWO). Q.Y. thanks the China Scholarship Council for financial support. We would like to thank Dr. Jun Gao for performing the Raman spectroscopy measurements.

\section{REFERENCES}

TG. Li, A. Luican, J. M. B. Lopes dos Santos, A. H. Castro Neto, A. Reina, J. Kong, and E. Y. Andrei, Nat. Phys. 6, 109 (2010).

${ }^{2}$ I. Brihuega, P. Mallet, H. González-Herrero, G. Trambly de Laissardière, M. M. Ugeda, L. Magaud, J. M. Gómez-Rodríguez, F. Ynduráin, and J.-Y. Veuillen, Phys. Rev. Lett. 109, 196802 (2012).

${ }^{3}$ E. Suárez Morell, J. D. Correa, P. Vargas, M. Pacheco, and Z. Barticevic, Phys. Rev. B 82, 121407(R) (2010).

${ }^{4}$ Y. Cao, V. Fatemi, S. Fang, K. Watanabe, T. Taniguchi, E. Kaxiras, and P. Jarillo-Herrero, Nature 556, 43 (2018).

${ }^{5}$ W. Yan, M. Liu, R.-F. Dou, L. Meng, L. Feng, Z.-D. Chu, Y. Zhang, Z. Liu, J.-C. Nie, and L. He, Phys. Rev. Lett. 109, 126801 (2012).

${ }^{6}$ A. Luican, G. Li, A. Reina, J. Kong, R. R. Nair, K. S. Novoselov, A. K. Geim, and E. Y. Andrei, Phys. Rev. Lett. 106, 126802 (2011).

${ }^{7}$ S. J. Ahn, P. Moon, T.-H. Kim, H.-W. Kim, H.-C. Shin, E. H. Kim, H. W. Cha, S.-J. Kahng, P. Kim, M. Koshino, Y.-W. Son, C.-W. Yang, and J. R. Ahn, Science 361, 782 (2018).

${ }^{8}$ M. Yankowitz, S. Chen, H. Polshyn, Y. Zhang, K. Watanabe, T. Taniguchi, D. Graf, A. F. Young, and C. R. Dean, Science 363, 1059 (2019).

${ }^{9}$ Y. Cao, V. Fatemi, A. Demir, S. Fang, S. L. Tomarken, J. Y. Luo, J. D. SanchezYamagishi, K. Watanabe, T. Taniguchi, E. Kaxiras, R. C. Ashoori, and P. Jarillo-Herrero, Nature 556, 80 (2018).

${ }^{10}$ B. Padhi, C. Setty, and P. W. Phillips, Nano Lett. 18, 6175 (2018).

${ }^{11}$ L. A. Gonzalez-Arraga, J. L. Lado, F. Guinea, and P. San-Jose, Phys. Rev. Lett. 119, 107201 (2017).

${ }^{12}$ A. L. Sharpe, E. J. Fox, A. W. Barnard, J. Finney, K. Watanabe, T. Taniguchi, M. A. Kastner, and D. Goldhaber-Gordon, Science 365, 605 (2019).

${ }^{13}$ R. Bistritzer and A. H. MacDonald, Proc. Natl. Acad. Sci. U. S. A. 108, 12233 (2011).

${ }^{14}$ P. San-Jose and E. Prada, Phys. Rev. B 88, 121408 (2013).

${ }^{15}$ S. Huang, K. Kim, D. K. Efimkin, T. Lovorn, T. Taniguchi, K. Watanabe, A. H. MacDonald, E. Tutuc, and B. J. LeRoy, Phys. Rev. Lett. 121, 037702 (2018).

${ }^{16} \mathrm{~S}$. G. Xu, A. I. Berdyugin, P. Kumaravadivel, F. Guinea, R. K. Kumar, D. A. Bandurin, S. V. Morozov, W. Kuang, B. Tsim, S. Liu, J. H. Edgar, I. V. Grigorieva, V. I. Fal'ko, M. Kim, and A. K. Geim, Nat. Commun. 10, 4008 (2019).

${ }^{17}$ H. Polshyn, M. Yankowitz, S. Chen, Y. Zhang, K. Watanabe, T. Taniguchi, C. R. Dean, and A. F. Young, Nat. Phys. 15, 1011 (2019).

${ }^{18}$ H. Yoo, R. Engelke, S. Carr, S. Fang, K. Zhang, P. Cazeaux, S. H. Sung, R. Hovden, A. W. Tsen, T. Taniguchi, K. Watanabe, G. Yi, M. Kim, M. Luskin, E. B. Tadmor, E. Kaxiras, and P. Kim, Nat. Mater. 18, 448 (2019).

${ }^{19}$ P. Rickhaus, J. Wallbank, S. Slizovskiy, R. Pisoni, H. Overweg, Y. Lee, M. Eich, M. Liu, K. Watanabe, T. Taniguchi, T. Ihn, and K. Ensslin, Nano Lett. 18, 6725 (2018).

${ }^{20}$ A. C. Ferrari, J. C. Meyer, V. Scardaci, C. Casiraghi, M. Lazzeri, F. Mauri, S. Piscanec, D. Jiang, K. S. Novoselov, S. Roth, and A. K. Geim, Phys. Rev. Lett. 97, 187401 (2006).

${ }^{21}$ M. Yankowitz, J. Xue, D. Cormode, J. D. Sanchez-Yamagishi, K. Watanabe, T. Taniguchi, P. Jarillo-Herrero, P. Jacquod, and B. J. LeRoy, Nat. Phys. 8, 382 (2012).

${ }^{22}$ Q. Yao, R. van Bremen, G. J. Slotman, L. Zhang, S. Haartsen, K. Sotthewes, P. Bampoulis, P. L. de Boeij, A. van Houselt, S. Yuan, and H. J. W. Zandvliet, Phys. Rev. B 95, 245116 (2017).

${ }^{23}$ J. M. B. Lopes dos Santos, N. M. R. Peres, and A. H. Castro Neto, Phys. Rev. Lett. 99, 256802 (2007).

${ }^{24}$ I. Martin, Ya. M. Blanter, and A. F. Morpurgo, Phys. Rev. Lett. 100, 036804 (2008).

${ }^{25}$ L. Ju, Z. Shi, N. Nair, Y. Lv, C. Jin, J. Velasco, Jr., C. Ojeda-Aristizabal, H. A. Bechtel, M. C. Martin, A. Zettl, J. Ananlytis, and F. Wang, Nature 520, 650 (2015).

${ }^{26}$ L.-J. Yin, H. Jiang, J.-B. Qiao, and L. He, Nat. Commun. 7, 11760 (2016). 